\title{
Macht, Raum, Geschlecht? Perspektiven auf gegenwärtige Politiken ${ }^{1}$
}

\author{
Mit Mechthild Bereswill, Ines Kappert und Simona Pagano. \\ Moderiert von Mart Busche und Felix Krämer
}

Felix Krämer: Wir freuen uns, dass Sie so zahlreich noch da sind zur Podiumsdiskussion. Wir wollen uns ganz kurz vorstellen, die wir das moderieren. Ich bin Felix Krämer, ich arbeite zur Nordamerikanischen Geschichte in Erfurt.

Mart Busche: Ich bin Mart Busche, ich bin assoziiert gewesen am Graduiertenkolleg und promoviere zu Nicht-Gewalttätigkeit und Männlichkeit und bin jetzt an der Alice Salomon Hochschule in Berlin tätig.

Felix Krämer: Wir wollen versuchen, das auf der Tagung Gehörte jetzt auf unserer Podiumsdiskussion in Korrespondenz mit einer Gegenwart - auch durchaus mit einer politischen Gegenwart - zu bringen. Man könnte sagen, wir versuchen eine Art Live-Schalte zwischen den vielfältigen interdisziplinären Forschungen, die im Kolleg in den vergangenen Jahren durchgeführt wurden und ihren politischen Implikationen auf aktuelle Verkörperungen,

1 Die Diskussion wurde geführt als Abschlussveranstaltung der Tagung „Interdisciplinary Matters: Doing Space while Doing Gender. Neue Perspektiven auf Materialität, Medialität und Temporalität“, die am 28. und 29. Juli 2016 an der Georg-August-Universität Göttingen stattfand. 
beziehungsweise Verräumlichungen von Geschlecht und sexuellen Identifikationen.

Mart Busche: Dazu wollen wir überlegen, was politische Implikationen von Raum und Geschlecht denn eigentlich sein können und verschiedene Dinge ansprechen. Wir werden nicht alles diskutieren können; keine Ahnung, ob schon mal jemand die Gender-Dimension des Brexits erforscht hat. Worüber wir sicherlich reden werden, sind die Migrationswege, die auch durch die populistischen Bewegungen, die es gerade gibt, beeinflusst sind, und was das mit Geschlechterverhältnissen zu tun hat. Wir können darüber reden, wieso zwar die Sexualität des Attentäters von Orlando Thema ist, der dort einen Gay Club als Ziel wählte, sein Geschlecht aber seltsamerweise unbesprochen bleibt.

Eine zentrale Person unserer Programmplanung ist heute nicht hier, dazu werde ich gleich noch was sagen. Die hätten wir gefragt: „,Was ist eigentlich das Internet für ein Raum, wenn quasi über Nacht so schwierige Themen wie Alltagssexismus am Beispiel des \#aufschrei thematisiert werden oder auch der Staatsrassismus in den USA am Beispiel von \#BlackLivesMatter eine immense Aufmerksamkeit erfährt? Internet bzw. die reale Welt, das sind vielleicht auch mehrere Räume, die das tatsächliche Veränderungspotenzial vielleicht auch kontingent erscheinen lassen, je nachdem, wieviel reale Mobilisierung dann in die reale Welt überschwappt. Den Unterschied bemerken Menschen ja dann, wenn tatsächliche Demonstrationen unter den Motti dieser Hashtags, die sonst auf Twitter erscheinen, stattfinden. Leider musste Jasna Strick, das ist eine Mitinitiatorin des Hashtags \#aufschrei, sowie auch des Hashtags \#ausnahmslos, ihre Teilnahme heute absagen. Wir hatten sie als Netzfeministin eingeladen. Die Rolle werden wir jetzt so ein bisschen mit übernehmen, sofern wir das können. Das wird sehr begrenzt bleiben.

Felix Krämer: Aber nicht zu vergessen, freuen wir uns natürlich mit den Gästen, die wir haben, diskutieren zu können. Wir sind glücklich, dass wir einen so vielfältigen Kreis an Leuten hier haben, die sich wissenschaftlich und politisch im Feld solcher Dynamiken bewegen. Ich freue mich sehr, Ines Kappert begrüßen zu dürfen. Sie leitet seit vergangenem Jahr das GundaWerner-Institut für feministische Studien der Böll-Stiftung. Zuvor hat sie als Journalistin gearbeitet und das Meinungsressort der taz geleitet. Sie hat ein 
Buch zu Männlichkeitskrisendiskursen als Kapitalismuskritik in der Mainstream-Kultur geschrieben und beschäftigt sich momentan unter anderem mit Eckensteher-Diskurssträngen. Wenn ich das schon mal so zusammenfassen darf: Es geht um Männlichkeit, Rassismus, sowie die Verschränkung von frauenpolitischen Argumenten mit Xenophobie.

Mart Busche: Ebenfalls freuen wir uns, Simona Pagano begrüßen zu dürfen, die in unserer Runde hier dabei ist. Sie hat an der Europa-Universität Viadrina Interkulturelle Kommunikation mit den Schwerpunkten Transdisciplinary Gender Studies, Migration/Ethnicity/Ethnocentrism studiert und im EU-Projekt „TOLERACE-The semantics of tolerance and (anti-)racism in Europe“" gearbeitet. Sie forscht im Kolleg zu italienischen Camps, in denen Rom_nja leben. Die Rede vom Flüchtlingsstrom und Lagern als Raum an und hinter den Grenzen hat nach Beginn ihrer Forschung womöglich eine neue Bedeutung bekommen. Darüber wollen wir auch sprechen.

Felix Krämer: Und wir freuen uns, dass Mechthild Bereswill bei uns ist. Sie ist Geschlechtersoziologin aus unserem Kollegskreis. Und neben ihrer Beschäftigung mit Gender, sozialer Kontrolle, totalen Institutionen - was sicherlich tief in die Stratifizierung auch sozialer Räume ragt - kann ich als Postdoc der letzten Stunde aus vielen Kollegskontexten sagen, wie andere sicher auch, dass ihr interdisziplinärer Scharfsinn und ihre theoretischen Ideen unser Kolloquium immer bereichert haben. Und deswegen freuen wir uns auch, dass wir mit Mechthild Bereswill eine Brücke zum Tagungsthema „interdisciplinary matters“ haben, und erhoffen uns in der Diskussion, die Projekte und Ideen der vergangenen Jahre in Bezug zur Gegenwart zu setzen.

\section{GENDER-DISPOSITIV}

Mart Busche: Wir wollen jetzt so vorgehen, dass wir die Diskussionsrunde erstmal von der Raumseite und dann von Geschlechterseite aus nach Machtwirkungen und politischen Implikationen fragen. Im Anschluss wollen wir gerne beide Perspektiven, Raum und Geschlecht - Macht wirkt natürlich immer zwischen ihnen - in der gemeinsamen Diskussion mit dem Plenum verknüpfen. Wir würden zuvor nun noch ein paar einleitende Bemerkungen machen, nämlich den Begriff „Gender-Dispositiv“ einführen bzw. auch gerne 
zur Disposition stellen und gemeinsam besprechen, warum wir uns möglicherweise innerhalb eines Gender-Dispositivs bewegen und bewegen müssen. [EINLEITUNG]

Felix Krämer: So ein Dispositiv hat ja historisch eine gewaltige Ausdehnung und systematische Forschungen können ausloten, wo und warum wir uns gegenwärtig an so einem Punkt in der Geschlechterforschung befinden. Und daher rührt unsere Überlegung, dass es möglicherweise hilfreich wäre, einen an Foucault orientierten Dispositivbegriff hörbar zu machen, um die vielfältigen zeiträumlichen Facetten des Machtapparats zu greifen, innerhalb dessen Körper, Diskurse und Praktiken mobilisiert werden und organisiert sind. Die Geschlechterarrangements der Gegenwart sind eben nicht nur lose Diskurse, sondern produzieren möglicherweise einen gewaltigen „Körper von Gewicht“, innerhalb dessen neoliberale Verteilungslogiken operieren, innerhalb dessen wir denken, sprechen und agieren müssen und innerhalb dessen Menschen sowohl verdeckt werden als auch sprachlos bleiben, obwohl sie darin organisiert sind - diese Ambivalenz gilt es herauszuarbeiten. Möglicherweise kann ein Dispositiv „Gender“ - und das ist ein Spezifikum unserer Fragestellung - demzufolge auch nur verortet werden, indem man die Machtstrukturen in ihrer zeiträumlichen Dimension versteht. Betrachtet man also die Frage in unserer Diskussion zu den gegenwärtigen Geschlechterräumen aus der Perspektive „Macht, Raum, Geschlecht?“ dann spiegeln sich die Suchbewegungen nach Materialität, Medien, Ordnungen, die Brüchigkeiten, die wir im Verlauf unserer Tagung gesehen haben, in den inspirierenden Analysen in der Gegenwart. Und wir haben sozusagen die wissenschaftliche Suche nach den Strukturen und Ausformungen eines solchen Gender-Dispositivs gesehen. Das wäre die Arbeitshypothese und jetzt wollen wir sehen, wie sich das mit gegenwärtigen Politiken verschalten lässt.

Mart Busche: Hierzu ist es möglicherweise hilfreich, das eben als multi-relationales Gebilde, als einen Machtkomplex oder auch mit dem Männlichkeitsforscher Edgar Forster gesprochen als einen „Wahrheitsraum“, also einen Raum, in dem seit Jahrhunderten nach Wahrheit gesucht wird, zu fassen. Wenn man das so betrachtet, dann sieht man auch, dass es da nicht immer um emanzipatorische Bewegungen ging, sondern dass Gender selbst zum umkämpften Ort geworden ist, wo manche innerhalb dieses Gender-Dispositivs genau das Gegenteil von emanzipatorischen Strategien produzieren, 
nämlich Grenzen, Begrenzungen, Abwertungen, oder möglicherweise auch einfach apolitische Orte. Und andere - wiederum sehr konkret - zum Beispiel Forschungszentren gründen, sich vernetzen, um explizit nach Strategien zu suchen, um durch Wissenschaft in Öffentlichkeits- und Wahrheitspolitiken zu intervenieren. Wobei möglicherweise dann einiges Andere wieder hinten runter fällt.

Felix Krämer: Im Zusammenhang mit so einem Dispositiv stellt sich dann notwendigerweise die Frage nach Macht, die sich nach einer theoretischen Erörterung im Hinblick auf Raumpolitiken vielleicht auch runterbrechen lässt, auf die Frage, wer in der politischen Öffentlichkeit mit welchen Interessen was verfolgt und warum. Zweitens organisiert das Dispositiv wie und wo etliche Gruppen und Leute zu hören sind bzw. andere in diesem Resonanzraum eben nicht sprechen können und unhörbar bleiben. Es lenkt Verteilungen und Sichtbarkeiten, produziert Hegemonien oder festigt subalterne Verhältnisse. Wir wollen hier die Machtfrage zuerst mit der Perspektive auf Räume aufrollen, und da wäre die erste Perspektive: In welchen Räumen entfalten sich welche vergeschlechtlichten Dynamiken und wie werden Räume strukturierend tätig? Während Ordnungsmuster zwischen öffentlich und privat, Führungsfunktionen im öffentlichen Bereich und Fürsorgearbeit - auch in ökonomischer Unscheinbarkeit - bekannt sind, sind eben dritte halböffentliche, mediale, virtuelle, andere Räume hinzugekommen, die neue Dynamiken und Risiken und vielleicht auch Freiräume und Spielräume bieten. Bevor wir im zweiten Block also dann genauer schauen, welche rechten Beanspruchungen auf den Genderbegriff wir mit welchen Implikationen ausmachen können, würden wir im ersten Block jetzt gerne ausloten, welche Raumfigurationen auch von Machtbeziehungen gekennzeichnet sind.

\section{FEMINISMUS}

Mart Busche: Was kann die Raumperspektive zum aktuellen Stand von Geschlecht und Geschlechterpolitiken beitragen? Welche notwendig politischen Räume, innerhalb derer wir forschen und leben, sind auszumachen? Das wäre jetzt erstmal unsere Leitfrage und unser erster Punkt darin heißt Feminismus. 
Felix Krämer: Dazu würden wir gerne Mechthild Bereswill befragen. Der Vortrag von Imke Schmincke hat uns zu Beginn als zeitgeschichtliche Klammer in die Bewegung eingeführt, innerhalb derer Geschlecht problematisiert wurde. Was unter westlichem Feminismus der zweiten Frauenbewegung zu verstehen ist, kam da in Bewegung. Welcher Raumbegriff ist aus Ihrer Sicht in der Forschungsperspektive von Imke Schmincke angesprochen worden?

Mechthild Bereswill: Okay, das war ein Fragezeichen, ich darf jetzt anfangen. Bevor ich jetzt sofort eins zu eins auf die Frage antworte, mache ich selbst noch mal eine kurze Vorbemerkung zu meiner Perspektive auf diese und auch dann die folgenden Fragen. Und ich will mich zunächst auch bedanken, dafür dass ich jetzt hier mitwirke und auch für diese - wie ich finde - unglaublich gute Vorbereitung des Panels, weil ich die Chance hatte, mich mit den Fragen schon auseinanderzusetzen. Und weil ich ja wusste, dass ich mich jetzt zunächst mit der neuen Frauenbewegung auseinandersetzen soll und auch dem Impuls, den Imke Schmincke mit ihrem Beitrag hier und auch den anderen Vorträgen, die wir von ihr schon im GraKo gehört haben, gesetzt hat, ist es mir zunächst erstmal ein Anliegen, deutlich zu machen: Ich spreche jetzt darüber aus der Perspektive der Wissenschaflerin, ich bin aber auch eine Zeitzeugin. Und deshalb ist mein Blick auf die wissenschaftliche Auseinandersetzung mit der neuen Frauenbewegung teilweise sicherlich getrübt. Ich verliere dann immer mal den Abstand und denke: „Nee, das geht so gar nicht und so war das auch nicht“. Und das ist mir jetzt schon auch in anderen Kontexten widerfahren, wo eben Historiker_innen zur neuen Frauenbewegung geforscht haben. Teilweise waren da auch Kolleg_innen dabei, denen es ähnlich ging. Ich werde mich aber versuchen, obwohl ich Rollentheorie eher zu schlicht finde, jetzt mal auf die Rolle der Wissenschaftlerin zu beschränken. Und wenn Sie mich dann fragen, welche Raumperspektive oder welcher Raumbegriff uns mit auf den Weg gegeben wird in der Untersuchungsperspektive von Imke Schmincke, dann würde ich sagen - jetzt mal sehr, sehr allgemein - geht es ja in der Perspektive auf die neue Frauenbewegung in Westdeutschland um die radikale Infragestellung der Grenzen des politischen Raums. ${ }^{2}$ Und ich finde dann aber den Singular in der Formulierung

2 Vgl. auch Schmincke, Imke (2018): Subjektivierung und (Körper-)Politik. Zur Bildung des kollektiven Subjekts Frauenbewegung. In: Alkemeyer, Thomas/ 
auch schon falsch, weil die Auseinandersetzung mit dieser sozialen Bewegung und auch den angrenzenden sozialen Bewegungen - und das zeigen auch ihre Beispiele für mich deutlich: Es gibt jeweils einen politischen Ort, wie zum Beispiel diesen SDS-Kongress, aber dort entfalten sich sofort auch verschiedene Räume. Und in welchem Verhältnis diese Räume zueinander stehen, wäre für mich auch noch eine weitere spannende Frage. Die Heinrich-Böll-Stiftung, in der ich selbst auch noch mit Gunda Werner zusammengearbeitet habe, hat in ihrer Entstehung ja mal einen Kongress veranstaltet, der hieß: „Wie weit fliegt die Tomate?“- Das ist ja auch ein räumliches Bild, also wieviel Strecke hat diese Tomate gemacht. Und daran musste ich denken, auch bei dieser süffisanten Beschreibung aus dem Spiegel. Ich denke, dass es sehr fruchtbar ist, genau mit einer Raumperspektive auf diese Konfliktdynamiken auch zu schauen, und zwar in mehrfacher Hinsicht: Sowohl auf den größeren politischen Raum, in dem sich das abspielt, zu dem ich eben auch vorschlagen würde, ihn noch stärker heranzuziehen (dazu sage ich gleich auch noch an einem Beispiel etwas) - als auch in der immanenten Auseinandersetzung. Und diese Beschäftigung sollte von vornherein mit ansetzen, weil auch die neue Frauenbewegung natürlich kein homogener Raum war. Es wird ja aus heutiger Sicht immer gesagt, und das ist ja auch eine richtige Kritik, dass da irgendwie an einem einheitlichen Subjekt Frau gestrickt worden sei. Ich würde sagen, dass immer beides parallel stattgefunden hat: Eine ganz starke Auseinandersetzung mit Unterschieden und Gemeinsamkeiten. Allerdings hat sich dann im hegemonialen Diskurs ganz oft dieses Sprechen ,als Frau“ durchgesetzt, als eine politische Strategie, obwohl es dies aber nie in einem homosozialen Raum ,neue westdeutsche Frauenbewegung“'gegeben hat. Das finde ich eine irreführende Betrachtung. Dies hat Imke Schmincke auch nicht gemacht. Ich kommentiere jetzt auch nicht den Vortrag - das ist mir auch noch ein Anliegen - ich nehme den eher als Ausgangspunkt, um noch ein paar weitere Überlegungen anzubieten. Im Mittelpunkt des Vortrags standen ja die Körperpolitiken der Frauenbewegung und das finde ich einen superinteressanten Ankerpunkt für eine weitere Auseinandersetzung. Wenn ich das jetzt nochmal auf die Frage der raumtheoretischen Reflektion beziehe, dann fände ich es ausgesprochen interessant, sich tatsächlich mit dem Körper auch als Innenraum zu beschäftigen. Wenn wir

Bröckling, Ulrich/Peters, Tobias (Hg.): Jenseits der Person. Zur Subjektivierung von Kollektiven, Bielefeld: transcript Verlag, S. 133-149. 
wirklich die Praktiken der Frauenbewegung, der Gruppen, die Körperpolitiken gemacht haben in den Frauenbewegungen betrachten - und da ging es ja nicht mehr nur um Westdeutschland, sondern auch um die internationalen Dimensionen - dann wäre es aus meiner Sicht sehr lohnenswert, die Quellen, die vorliegen - und auch die Praktiken und auch Zeitzeug_innen-Interviews würde ich hierzu für sehr sinnvoll halten - tatsächlich daraufhin zu untersuchen, wie der Körper wirklich als ein Innenraum konstruiert wurde - gerade als ein Gegenentwurf in der Zeit. In den 1960er und auch bis in die 1970er Jahre hinein wurde in den rigiden Körperpolitiken der westdeutschen Nachkriegsgesellschaft der weibliche Innenraum sehr stark auch gewaltförmig untersucht. Ein drastisches Beispiel aus meiner eigenen Forschung ist, dass wir in den Akten der Mädchen, die in den Erziehungsheimen untergebracht waren, routinemäßige Virginitätsprüfungen nach Entweichungen finden. Wir sehen, dass es da ganz starke invasive Verfahren in Auseinandersetzung mit dem sittlich abweichenden und sittlich gefährdeten weiblichen Körper gibt. Und ich erwähne dieses Beispiel an der Stelle, weil ich auch anregen würde, die Körperpolitiken der Frauenbewegung in Relation zu den Körperpolitiken der politischen Situation zu untersuchen, in der die Frauenbewegung agiert hat - also eine Relation auszumachen, das hielte ich für eine sehr fruchtbare Perspektive.

Nun habe ich schön artig auf die Raumfrage geantwortet. Ich habe heute Morgen allerdings überlegt, ob ich eher ein bisschen provozierend sagen sollte: „Es muss ja nicht alles mit Raum untersucht werden. Was ist der Gewinn, wenn wir auf soziale Bewegungen raumtheoretisch schauen?" Da bin ich tatsächlich ambivalent, was der Gewinn ist. Und da würde ich mir dann auch eher so eine etwas vage Antwort selbst geben und sagen: „Das kommt dann tatsächlich auch auf die Theorieperspektive an, die wir einnehmen."Ich bin zum Beispiel nicht so überzeugt davon, dass der relationale Raumbegriff von Martina Löw für eine soziale Bewegungsforschung so viel weiterträgt. Wenn wir jetzt Zeit hätten, könnten wir überlegen, ob wir Lefebvre anschauen und sagen: Vielleicht ist doch dieses Ebenenmodell, was auch stärker rückgebunden ist, tatsächlich auch an institutionelle verfestigte Kontexte, auch ein interessantes Modell. Aber das ist eher ein kleiner Ausflug. [EINLEITUNG]

Und als letzte Anmerkung, weil es natürlich bei diesem Podium für mich aus meiner Perspektive auch zurückblickend auf meine eigene Biografie und 
die aktuelle Position im Wissenschaftssystem natürlich immer um das Verhältnis von Wissenschaft und Politik geht - das rufen Sie natürlich mit dieser Panel-Themensetzung auch auf - würde ich sagen, wenn wir die Frauenbewegungen auch als Wissensräume untersuchen wollen, dass dann für die westdeutsche Frauenbewegung die Schriften der Frauensommeruniversitäten eine ungeheuer interessante Quelle wären. In ihnen könnten wir sehen, wie dort der Raum konstruiert wurde. Die erste Publikation der Frauensommeruniversität wird damit eröffnet - ich bin mir aber nicht sicher, aber ich glaube es ist Karin Bock -, dass eine Autorin schreibt: „Das ist hier eine Bildungsveranstaltung für alle Frauen. "Und da haben wir wieder dieses Kollektivsubjekt Frau. Aber wir haben natürlich gleichzeitig, wenn wir von heute aus drauf gucken, auch einen inklusiven Raum für Personen, die nicht aus dem akademischen Milieu kommen. Das war in den Anfängen der Frauenforschung ja auch eine der heftigsten Debatten: Parteilichkeit, Überwindung bestimmter Grenzziehungen, Öffnung von sozialen Räumen, partizipative Forschung. Und da finde ich tatsächlich die Schriften der Frauenuniversitäten eine ganz spannende Quelle, um sich mit diesen Konstruktionen eines Wissensraums auseinanderzusetzen.

\section{MEDIEN}

Felix Krämer: Ich würde gerne im Anschluss daran eine institutionelle Frage an Ines Kappert stellen. Sie haben ja im Meinungsressort der taz gearbeitet. Wo, würden Sie sagen, sind die Chancen und Grenzen von Printjournalismus für emanzipative, feministische oder queere Politiken? Was ist das für ein medialer Raum? Gleich im Anschluss könnte ich die Frage auch damit verbinden, was die politische Codierung von so einem Institut wie dem GundaWerner-Institut für eine Ausgangslage darstellt für politische Intervention in gegenwärtige Politiken und was das für ein institutioneller Raum ist, im Gegensatz etwa zu Universitätslandschaften oder auch den universitären Kollegs.

Ines Kappert: Ich bin ja hier gecastet, als jemand, die inzwischen schon politische Bildungsarbeit macht - keine Wissenschaft mehr - und eben vorher das Meinungsressort geleitet hat als Redakteurin, also acht Jahre lang im Journalismus, im Printjournalismus war. Welche Interventionsmöglichkeiten 
gibt es im Printjournalismus? Man muss sagen - jetzt generell gesprochen, über die taz hinaus gesprochen - gibt es keine feministische Berichterstattung. Die ganzen Frauenseiten, die es mal gab, also in den Medien, in denen es Frauenseiten mal gegeben hat, die sind alle aufgelöst worden. Unter anderem mit dem Argument: „Wir reden jetzt eben nicht mehr von Frauen, wir reden von Gender und damit ist sozusagen die Ghettoisierung auf einer Seite eben nicht mehr adäquat." Das hat de facto aber dazu geführt - und ich würde mich dieser Argumentation theoretisch auch anschließen -, dass Gender komplett zurückgedrängt wurde. Wenn man sich die Redaktionen anguckt, kann man wirklich ganz, ganz, ganz platt werden - und an der Stelle ist auch plumpes Denken adäquat: Die Redaktionen werden zu $90 \%$ von Männern geleitet. Und zwar sowohl in der Politik als auch in der Kultur. In der Kultur ist es sogar noch einen Tick schlimmer, wenn man diese Art von Monokultur kritisiert. Das hat auch Auswirkungen auf die Berichterstattung, weswegen zum Beispiel in der Süddeutschen Zeitung oder auch in der FAZ, also in unseren Qualitätsprintmedien, so gut wie überhaupt kein Feminismus stattfindet. Wenn das Thema aufkommt, dann geht es um Vergewaltigung, weil das natürlich enorm skandalisiert - „,ex und crime“ - das wird eher aus dieser Logik heraus zum Thema. Und man hat es auch gesehen, dass die Auseinandersetzung jetzt mit der Reform des Sexualstrafrechts, die ja gerade das Politikum war, dass es eben nur Meinungsäußerungen gab, aber eigentlich so gut wie keine Berichterstattung, die einfach mal die Komplexität aufgedröselt hätte. Bei der taz war es ein bisschen anders, weil ich bei der taz einfach machen konnte, was ich wollte. Das ist eben das Prinzip taz, da gibt es keinen Herausgeber, da ist jeder seine eigene Chefin. Insofern konnte ich auf den Meinungsseiten das durchsetzen und habe es auch durchgesetzt. Aber das ist eben auch ein Sonderfall.

Jetzt im Vergleich: Was sind die Unterschiede zwischen Journalismus und politischer Bildungsarbeit? Ich arbeite jetzt ja in einem Institut, dessen dezidierte Aufgabe ist, Feminismus und Geschlechterdemokratie zu vermitteln. Also eben nicht feministische Studien zu betreiben - wir machen keine Wissenschaft, sondern wir sollen Inhalte an ein akademisch vorgebildetes Publikum vermitteln. Und da ist es so - ich kann das jetzt gar nicht so normativ sagen, ich kann das nur einfach empirisch sagen -, dass das, was mich gerade sehr interessiert, die Rassismen innerhalb der feministischen Diskurse sind, die halt auch im grünen Umfeld existieren. Wir haben uns stark im Gunda-Werner-Institut mit Köln auseinandergesetzt, sicherlich auch eine 
Metapher für Raum-Macht-Geschlecht. Sie wissen das ja alle, dass Köln zur Chiffre wurde dafür, dass ,der fremde Mann“ der Triebtäter ist, dass ,der fremde Mann“ die Gefahr oder die Gefährdung „der eigenen Frau“ im öffentlichen Raum bebildert, das Sinnbild dafür wird. [TAHRIRPLATZ] Das Sexualstrafrecht, das jetzt ja im feministischen Sinne reformiert worden ist, worin sexuelle Selbstbestimmung jetzt zum ersten Mal als Rechtsgut tatsächlich etabliert ist, ist erkauft worden mit erleichterten Ausweisungen. Dies ist eins zu eins verschränkt worden. Der Skandal, den wir in Köln hatten, nach der Berichterstattung, hat sich jetzt fortgesetzt in der Gesetzgebung und der feministische Aufschrei darüber blieb aus. Mit Ausnahme von eben den Frauen, die mit dem Aufruf \#ausnahmslos gesagt haben: „Es geht nicht um die Täter, es geht um die Tat, wir fordern eben die ausnahmslose Strafverfolgung." Die haben dann auch wiederum das Gesetz eben als rassistisch, also als im feministischen Sinne gut, im rassistischen Sinne schlecht kritisiert. Ansonsten passierte da nichts. Auch im grünen Umfeld passierte da nichts. Und ich denke, das ist etwas, wo es auch meine Aufgabe ist, weil es jetzt eben das Institut gesagt hat, daran weiter zu arbeiten, weil Rassismus immer mit Sexismus einhergeht und weil Feminismus, der rassistisch argumentiert, kein Feminismus ist.

Mart Busche: Vom journalistischen und Bildungsraum zur Uni - die Universität ist ja auch eine Bildungsinstitution, wenn man so will - und wir sind ja heute alle hier. Dabei wird diese Institution ja durchaus auch angefeindet und zwar gerade auch durchaus auf journalistischen Plattformen. Ich will es jetzt nicht Medien nennen, es sind Plattformen, wo gesagt wird, es werde „Gender-Hokuspokus“ gemacht und das durchaus auch mit so einem anti-intellektualistischen Habitus. Vielleicht stehen wir da heute, aber ich würde Sie gerne, Frau Bereswill, nochmal fragen - vielleicht nicht, ob Sie immer machen durften, was Sie wollten in der Wissenschaftslandschaft - aber, wenn man sich diese Forschungslandschaft, diese Topografie aus Elementen anschaut in der Wissenschaft, innerhalb derer die Gender Studies ein Element sind, auch die Geschlechtersoziologie und die Geschlechterforschung, die jetzt ja noch nicht so wahnsinnig alt sind: Können Sie ein paar Zäsuren setzen in der Entwicklung, wie das entstanden ist, welche Räume aufgegangen sind, welche Räume sich geschlossen haben? Was könnte da heute für uns interessant sein? 
Mechthild Bereswill: Okay, ich nehme erstmal das Bild der Landschaft auf, das ist mir zu malerisch. Ich würde an der Stelle wirklich mal von Strukturen sprechen. Es handelt sich bei der Universität um eine Organisationsstruktur, mit bestimmten institutionell verfestigten Regeln und Regelwerken, sowohl informellen, als auch ganz formalen Regelwerken. Und es handelt sich um eine riesige Verwaltungsbürokratie. Nur, damit irgendwie klar ist, dass die Frage, wer kann machen, was sie oder er will, sich in diesem Kontext quasi platziert. Wobei ich es für völlig unproduktiv halte, so einen Kontrast aufzumachen zwischen der politischen Bildung und dem Journalismus als den Freien einerseits, die machen können, was sie wollen und jenen, die in den Wissenschaften irgendwie nur gespurt sind. Aus meiner Sicht gibt es in dieser bürokratischen Struktur nach wie vor viele Handlungsspielräume. - Das erstmal als eine allgemeine Vorbemerkung, auch unabhängig davon, ob ich mich in der Geschlechterforschung verorte oder nicht. Also: Es gibt Handlungsspielräume, aber die sind natürlich trotzdem quasi als Möglichkeitsräume mehr als gerahmt. Ich betone nochmal: - Bürokratie -, weil oft unterschätzt wird, dass eben tatsächlich bürokratisches Handeln ein Teil des Spiels ist im Umgang mit den Möglichkeitsräumen dieses Wissenschaftssystems.

Ich komme zur Frage nach der Öffnung und Schließung von Wissensräumen und zu den Zäsuren. Zu den Zäsuren - da durfte ich ja im Vorfelde auch schon darüber nachdenken - habe ich erst gedacht: Klar, Bologna, das wäre ja jetzt so eine Zäsur, die könnte ich jetzt gut mal nennen. Dann dachte ich: Nee, das mache ich jetzt mal nicht. Jetzt habe ich das genannt und nehme es dann gleich wieder zurück, weil ich das Bild der Zäsur zu stark finde. Das hat vielleicht auch etwas damit zu tun, zu welchem Zeitpunkt ich dann tatsächlich auf einer ordentlichen Professur im Wissenschaftsbetrieb angekommen bin, nämlich zu einem Zeitpunkt, als alle diese Weichen im Grunde schon gestellt waren. Meine eigene Biografie vor Bologna ist eben die des Weges hin zur Professur. Und daher bin ich immer vorsichtig, mit den Zäsuren, weil mein Blick natürlich ganz stark dann von jetzt und früher auch gekennzeichnet ist durch ganz unterschiedliche Positionen, die ich hatte. Ganz sicher ist die Einführung der modularisierten Studiengänge ein Mechanismus, an dem wir besonders gut Öffnungen und Schließungen von Wissensräumen beobachten können, aber das wäre genau zu untersuchen. Also da würde ich dann gerne genau hinschauen, weil ich glaube, dass das von Uni- 
versität zu Universität sehr unterschiedlich sein kann und auch von den jeweiligen Zuschnitten der Fächerkulturen und auch wenn wir sehen, wo die Gender-Studies-Studiengänge wie zusammengesetzt sind, ist es sicherlich unterschiedlich. Das Bild mit der Öffnung und Schließung von Wissensräumen ist ja ein Bild, was irgendwie natürlich dazu einlädt, zu sagen: „Das ist ambivalent, also weder öffnen die sich nur, noch schließen die sich“. Es ist eher die Frage nach einer tatsächlich gleichzeitigen Ungleichzeitigkeit in diesen Entwicklungen: Wo öffnen sich neue Räume?

Ein Graduiertenkolleg ist so ein Raum, der sich öffnet. Das heißt aber, dass wir uns gleichzeitig mit den Schließungsmechanismen, die durch die DFG und durch bestimmte Regeln vorgegeben sind, auch auseinandersetzen müssen. Und dass das natürlich auch Einfluss nimmt auf die Gestalt eines solchen Raums. Und das heißt, aus meiner Sicht hat die Frauen- und Geschlechterforschung, inklusive Männlichkeits- und Queer-Studien und allen Ansätzen, die mittlerweile da auch Einfluss nehmen, natürlich ganz stark neue Denkräume geöffnet und unglaublich viele Forschungsbefunde produziert. Es gibt eine riesige, unübersichtliche Menge von Forschungsbefunden, insbesondere in den Sozial- und Geisteswissenschaften, die durch diese Etablierung neuer Räume überhaupt erst möglich wurden und die dann auch wieder neue Denkräume schaffen. Und es ist gar nicht so leicht, die zu fassen zu kriegen, weil wir selbst hier auch immer nur einen Ausschnitt sehen. Insofern ist es aus meiner Sicht schon ziemlich wichtig in dem jeweiligen Kontext zu schauen, wo es tatsächlich weitere Handlungsspielräume und Entwicklungsmöglichkeiten gibt und wo sich auch Bedingungen schließen. Und ich bin außerdem der Meinung, dass wir selbst - ich jedenfalls nicht, so aus meiner ersten Person betrachtet -, dass wir das nicht vollumfänglich durchschauen. Bürokratien sind demnach aus meiner Sicht - auch da bin ich eben auch eine strukturkonservative Soziologin - genau deshalb so gebaut, weil sie uns irgendetwas abnehmen, was wir dann aber auch tatsächlich nicht mehr in vollem Umfang durchschauen. Insofern meine ich, wenn ich jetzt sagen würde, dass sich da was schließt und da was öffnet, dass das schon wieder Gegenstand eines gewissen Deutungsmusters wäre. Es ist ein Deutungsmuster, das dann schon wieder untersucht werden kann. Natürlich beobachte ich die Entwicklung und kann deshalb auch dazu Aussagen treffen, aber die sind eben immer auch standortgebunden. Das, was sich ganz stark in diesen Wissensräumen entwickelt hat, ist eben nicht nur eine ganz große Menge von Forschungsbefunden; sondern das, was für mich das enorme Potenzial dieser 
Forschungs- und Theorietradition ausmacht, ist, dass es sich fast durchgängig um erkenntniskritische Ansätze handelt. Und was für mich eben das Charmante an der Geschlechterforschung ist, ist die auf Dauer gestellte Selbstbeobachtung. Damit meine ich tatsächlich nicht Selbstbeobachtung im Sinne von Selbsterfahrung, sondern eigene Begriffe, Konzepte eigentlich immer in Bewegung zu halten und natürlich dann in so einem Raum wie einem solchen Graduiertenkolleg diese auch noch im interdisziplinären Gespräch wechselweise zu prüfen. Dass das seine Grenzen hat, das wissen wir alle, da muss ich jetzt nichts dazu sagen. Aber da wäre mir zum Beispiel auch der Begriff der Schließung dann zu stark, um das zu beschreiben.

Und ich habe dann als letzten Punkt zu dieser Frage danach, was sich öffnet und was sich schließt darüber nachgedacht, was es eigentlich bedeutet, dass an den meisten Universitäten die Verankerung der Gender Studies nach wie vor prekär ist. Es gibt zwar Zentren, aber wir alle wissen, dass es nicht überall feste Mitarbeiter_innenstellen gibt. Das ist eigentlich eine nach wie vor immer mit Unsicherheit verbundene Situation, die sich durch den hohen Drittmitteldruck, der eben zu diesen Mechanismen auch dazugehört, noch stärker in Richtung einer selbstorganisierten Absicherung entwickelt hat. Das wäre für mich eine Frage, wo wir in der Zukunft auch gucken müssen, wie sich da wieder neue Mechanismen von Öffnen und Schließen zeigen.

Mart Busche: ... auch wie sich das auf die feministische Theorieentwicklung auswirkt oder sich die eigene Positionierung dazu dann entwickelt. Da sprechen wir möglicherweise gleich nochmal darüber. Wir wollen jedoch zum nächsten Punkt kommen.

\section{GRENZ- UND MIGRATIONSRÄUME}

Felix Krämer: Wir haben den nächsten Punkt mit „Migrationsräume“ überschrieben und wollen von unterschiedlichen Seiten überlegen, was diesbezüglich Raum bedeuten könnte im politischen Zusammenhang der Gegenwart. Da würden wir gerne zunächst Simona Pagano fragen, was sich aus Raumperspektive der ,,border studies“ gerade im Nachdenken über Räume verändert. Es gibt den Begriff des „Grenzregimes“ - was ist das und wie kann das räumlich gefasst werden? 
Simona Pagano: Zunächst vielen herzlichen Dank für die Einladung hier auf das Podium. Der Begriff des Grenzregimes entsteht eigentlich so ein bisschen unter anderem als Gegenbegriff zum Begriff der „Festung Europa“ Mitte der 2000er Jahre, weil der Begriff der Festung Europa einen hermetisch abgeriegelten Raum impliziert, der unidirektional verläuft und in welchem Migrationsbewegungen gar nicht recht erfasst werden können. Daher wird auf den Begriff des Regimes zurückgegriffen, in dem Macht im Regimebegriff dezentral organisiert ist und der es erlaubt, eben die Agency, die Handlungsmacht der Migrant_innen mit zu fassen. Und dabei ist eben die Grenze kein fester, fixer Raum, sondern die Grenze ist, wie das Sabine Hess formulieren würde, eben ein „Kräfte- und Aushandlungsraum“. Oder Grenze ist ein soziales Verhältnis, also Grenze wird sozusagen in diesen Kämpfen, Reibungen und Kontingenzen produziert. In dem Sinne ist die Grenze auch gar nicht so fest lokalisierbar, sondern konkretisiert sich immer erst in verschiedenen Lokalisierungen. Es geht also nicht in erster Linie um eine territoriale Grenze, die fix ist, sondern Grenze als territoriale Linie - oder materialisiert in Grenzposten und -zäunen - ist kontingenter Teil und Effekt dieser Aushandlungen.

Mart Busche: Wie verschränkt sich denn deiner Meinung nach Geschlechterdifferenz politisch mit dem strukturellen Rassismus in Lagern, in Migrationsbewegungen? Du arbeitest ja zu Lagern und insbesondere zu Roma-Lagern in Italien. Kannst du vielleicht mal ein Beispiel aus deiner Forschung geben? Wir hatten ja heute schon mal den Begriff der Vulnerabilität gehört, der ja auch von Sergia Adamo genannt wurde, auch den Begriff der Agency - das taucht jetzt bei dir alles wieder auf. Vielleicht kannst du dazu etwas sagen. [EXPOSURE]

Simona Pagano: Ob und wie sich Geschlechterdifferenzen mit strukturellem Rassismus in der Etablierung von Camps niederschlägt, das versuche ich in meiner Forschung rauszufinden und ich bin dabei auch tatsächlich über den Begriff der Vulnerabilität hier und da gestolpert. In der Auseinandersetzung damit frage ich mich allerdings, inwiefern der Begriff fruchtbar gemacht werden kann oder nicht. Kurz zurück: Der Begriff taucht bspw. im Zusammenhang mit Risikoabwehr und Risikomanagement auf und ist hier ein Gegenbegriff zu dem der Resilienz. Er taucht auch im Zusammenhang mit der 
Kategorisierung von Gruppen auf, die als vulnerabel markiert werden, beispielsweise bei Naturkatastrophen unter anderem. Wenn diese Gruppen nicht mobil sein können, nicht migrieren können, dann werden sie als vulnerabel eingestuft. Kritisiert wurde der Begriff unter anderem im Rahmen des AntiTrafficking-Diskurses. In Anti-Trafficking-Kampagnen wurde gesagt: „Diese Frauen sind vulnerabel, deswegen müssen wir sie schützen“. Eine der Kritiken an dem Begriff lautet wiederum, dass der Begriff viktimisierend wirkt und Frauen eben Handlungsmacht abspricht. Das führt dann dazu, dass beispielsweise bestimmte Organisationen auf den Plan treten, um diese Frauen vor den Menschenhändlern zu retten. Eine andere, damit auch zusammenhängende Kritik bezieht sich auf den Umstand, dass im Rahmen dieses Vulnerabilitätsdiskurses auch versicherheitlichende Maßnahmen in Bezug auf Migration durchgeführt werden. Auch in meiner Forschung tritt der Begriff auf, und zwar werden in Italien Rom_nja insgesamt als vulnerable Gruppe kategorisiert. Diese Kategorisierung wird dann nochmal in Bezug auf Frauen, Frauen mit Kindern, alleinstehende Frauen, Alte, Behinderte und Obdachlose spezifiziert. Interessant ist, wie sich das verräumlicht äußern kann.

Ich habe an der Stelle ein Beispiel, und zwar von einer Frau, die mit ihrer Familie aus einem anderen europäischen Land nach Rom zieht und weil sie erstmal keine Bleibe hat, in ein Camp für Rom_nja geht. Zunächst leben sie bei der Schwiegermutter. Dann passiert folgendes: Der Mann wird verhaftet. Das Verhältnis zwischen Schwiegermutter und Schwiegertochter ist nicht gut. Sie kann daher nicht mehr bei der Schwiegermutter im Wohncontainer bleiben. Das heißt, sie fängt eine Art Odyssee in dem Camp an. Irgendwann ist sie in einem Zelt gelandet, was von der Verwaltung aus toleriert wird eigentlich darf man das nicht. Das Zelt brennt ab, wie es dazu kam, weiß man nicht. Dann wird ihr gesagt: „, Okay, pass auf, das geht zu weit, du kannst hier nicht bleiben, sondern kommst jetzt in ein Aufnahmezentrum nur für Roma“. Dort geht sie hin, hält es aber nicht aus. Es ist alles sehr eng, es gibt keine Fenster, es gibt keine Privatsphäre, die einzelnen Räume sind nur mit Vorhängen abgetrennt. Sie geht wieder zurück in das Camp, versucht es wieder mit einem Zelt. Auch an der Stelle wird ihr wieder gesagt, dass das mit dem Zelt nicht gehe. Daraufhin hat sie genug und besetzt einen Container. Diese Besetzung des Containers wird erneut toleriert. Es wird ihr als alleinstehender Frau mit Kindern dieser Schutzraum Container zugestanden. Irgendwann wird der Mann aus dem Gefängnis entlassen und geht wieder zur Familie 
zurück. Die familiäre Einheit ist wiederhergestellt. Noch ein entscheidender Einschub an dieser Stelle: Als diese Frau den Container besetzte, so sagte sie mir, habe sie ein Mitarbeiter der zuständigen Behörde angesprochen. Dieser habe sexuelle Gefälligkeiten als Gegenleistung für die Gewährung des Wohncontainers verlangt. Sie habe dies in jenem Moment entschieden abgelehnt. Ihr Mann kam also aus dem Gefängnis wieder zurück und die Familie war wieder vereint. Kurz darauf meldet sich die zuständige Behörde erneut und zwar um die Familie aus dem Container und dem Camp rauszuwerfen. Der Container wurde zerstört und die Famlie obdachlos.

Worauf ich hinauswill, ist, dass der Vulnerabilitätsbegriff sowohl vergeschlechtlicht als auch verräumlicht ist. Und zwar im Sinne einer Geschlechtszuschreibung und individuellen Eigenschaft von Personen: Als alleinlebende Mutter mit Kindern wird sie in jenem Zusammenhang für vulnerabel gehalten. In dem Augenblick, in welchem der Mann wieder zur Familie dazustößt, scheint die Vulnerabilität aufgehoben. In diesem Sinne würde ich schon bei der Kritik am Vulnerabilitätskonzept und seiner Instrumentalisierung mitgehen. Gleichzeitig würde ich aber eben den Begriff nicht gänzlich verwerfen, weil er ja trotz allem, worauf auch Judith Butler hinweist, eine Exposition Gewalt gegenüber benennen kann. [EXPOSURE] Und ich glaube, man kann den Begriff fruchtbar machen, indem man ihn in einem Machtverhältnis, oder in seiner Beziehung zu Machtverhältnissen verwendet, also beispielsweise in einer intersektionalen Analyse von Rassismus und Sexismus eben dort fruchtbar macht. Dann erkennt man, dass Vulnerabilität - verursacht durch Rassismus - überhaupt keine Schutzhandlung nach sich zieht. Diese Dimension bleibt ausgespart. Vulnerabilität bleibt hier, bezogen auf das eben genannte Beispiel, lediglich vergeschlechtlicht und zwar weiblich vergeschlechtlicht konnotiert.

Felix Krämer: Wir würden gerne gleich noch einen Schritt weitergehen in der Frage nach öffentlichen Räumen. Ich würde gerne Ines Kappert fragen: Sie haben sich ja mit dem, was ich vorher so ein bisschen schwammig als Eckensteher-Diskurs bezeichnet habe, beschäftigt, und ich würde gerne fragen, wie frauenpolitische Argumentationen sich diskursiv verschränken mit Männlichkeitsgesichtspunkten, hegemonialer Männlichkeit und mit diesem Diskurs um die ,,angry white men“. Wie spielt das zusammen und was würden Sie für eine Kritik zwischen Rassismus und kritischer Männlichkeitsperspektive platzieren? 
Ines Kappert: Der Begriff „Eckensteher“ wurde von Jan Fleischhauer geprägt, als Kolumnist von Spiegel Online. Es gibt sechs Kolumnist_innen, Margarete Stokowski ist dabei - kennen Sie vielleicht nicht, eigentlich ist das mit am öffentlichkeitswirksamsten, das sind so 6000 -Zeichen-Kolumnen, einmal pro Woche, das sind so die Klickmonster von SPON, also von Spiegel Online. Die haben eine hohe Reichweite, Sibylle Berg und eben auch Jan Fleischhauer. Jan Fleischhauer hat zu Anfang des Jahres gesagt: „Ja, das ist eben mit dieser Willkommenskultur doch alles nicht so prima und nicht so einfach, weil wir halt jetzt diese Eckensteherkultur haben“. Da wurde dieses Bild des nicht arbeitenden Asylsuchenden konstruiert oder des nicht arbeitenden schwarzhaarigen Mannes, der einfach so herumlungert, als mehr oder weniger latente Gefährdung - das knüpft dann auch wieder an Köln an -, der den öffentlichen Raum kriminalisiert und auch die Selbstbestimmung von Frauen gefährdet. Wobei natürlich immer die Frage ist, wer geschützt werden soll - um Vulnerabilität aufzugreifen: Die deutsche Frau soll geschützt werden! Diese Idee, dass die deutsche Frau oder die national zugehörige Frau vor dem Fremden geschützt werden soll, ist ganz klar ein kolonialistisches Konzept. Das gab es in den Kolonien, wo auch die Einheimischen nicht befugt waren, die Kolonialherr_innen in irgendeiner Weise zu belästigen und hat seine Fortsetzung im Nationalsozialismus gefunden. Sie erinnern sich ja vielleicht auch an das Bild, sowohl in der Süddeutschen als auch auf dem Focus-Cover, wo eine weiße Frau mit schwarzen Händen abgebildet war, beziehungsweise eine Schwarz-Weiß-Grafik, wo eine schwarze Hand in die Vagina der weißen, nackten Frau zeigt. Es gibt eine direkte Verbindung zu Nazi-Plakaten, wo die deutsche Frau aufgefordert wird, ihr Blut zu schützen und eben nicht die Rassenschande zu begehen und sich dann eben nicht entsprechend mit schwarzen Männern einzulassen.

Was ist jetzt daran interessant? Daran ist eben interessant, dass, ich sage es jetzt einmal unpolitisch, auf anachronistische Art und Weise ein weißer, heterosexueller Raum - ein männlich-heterosexueller Raum - konstruiert wird in Köln. [TAHRIRPLATZ] Es wird gesagt: „Das Problem sind die fremden Männer, die die weißen Frauen bedrohen“, unabhängig von dem, was da tatsächlich passiert ist. Dass da Frauen mit migrantischem Hintergrund auch sexuell angegriffen wurden, spielte in der medialen Berichterstattung beispielsweise so gut wie überhaupt keine Rolle. Es wurde nicht thematisiert. Es wurde zum Teil gezeigt, aber es wurde nicht problematisiert, es wurde nicht in den Diskurs aufgenommen. Und ich finde dieses Bild des 
Eckenstehers ist ein so derart gewalttätiger Begriff, weil unhinterfragt bleibt, was mit Menschen passiert, die hierherkommen, die vielleicht auch illegal hierherkommen und Asyl beantragen. Sie werden vom Arbeitsmarkt ausgeschlossen, der Familiennachzug ist ausgesetzt. Wir haben es hier mit verordneten Männerbünden zu tun, weil man ihnen nicht zugesteht, ihre Familien nachzuholen. Sie haben so gut wie keine Arbeitsmöglichkeiten, sie werden in Lager untergebracht. Die ganze Diskussion um dezentrale Unterbringung ist ja negativ beschieden worden, was heißt, dass ihnen kein Privatraum zugestanden wird. Dann sieht man sie in der Öffentlichkeit und sie werden als Kriminelle stigmatisiert. Und das korreliert mit einem Erstarken der Stimmen der „,white angry men“, wie Michael Kimmel das für die USA sehr gut analysiert hat. Das Sinnbild dafür ist jetzt natürlich Donald Trump, wo es so eine Machtinszenierung gab: „Ich sage, wer sich wo aufhalten darf. Ich erteile Sprechmacht. Und zwar nicht auf Grundlage irgendeiner Analyse oder einer Interessensvertretung, sondern einfach nur auf Grundlage dessen: Ich kann das! Ich kann ein- und ausgrenzen, weil ich es kann. Weil ich reich bin, weil ich dumm bin, weil ich männlich bin und weil ich aber diesen Machtraum zugesprochen bekomme." Und ich glaube, es ist ganz interessant sich das anzugucken, wie solche Bilder, die ja aus feministischer Perspektive völlig inakzeptabel sind, dann doch Einzug halten. [EXPOSURE] Also ich wiederhole mich jetzt, aber auch nur in einem Satz, hoffe ich: Es ist wirklich krass, wie die Islamophobie in feministischen Diskursen einfach mal so durchkam. Das ist eben nicht nur allein die Position von Alice Schwarzer, sondern sie wird von sehr, sehr, sehr, sehr, sehr, sehr vielen geteilt, die auch diese Idee haben, dass jetzt „der schwarzhaarige Mann“ tatsächlich den öffentlichen Raum und die sexuelle Selbstbestimmung von deutschen Frauen bedrohen würde. Damit beschäftigen wir uns.

Felix Krämer: Vielen Dank - also ein Plädoyer für hegemoniekritische und whiteness-kritische Perspektiven auch innerhalb dieser machtvollen Verschränkungen. Das ist ja auch etwas, das wir unter dem Stichwort Intersektionalität am Kolleg an unterschiedlichsten zeit-räumlichen Zusammenhängen immer wieder diskutiert hatten.

Ines Kappert: Was auch charakteristisch für diesen Diskurs ist: Frauen mit Migrationshintergrund kommen überhaupt nicht zu Wort. Es gibt natürlich auch eine Patriarchatskritik genau von dieser Seite und das wird ebenfalls 
ausgegrenzt. Zum Sprechen autorisiert werden eben ,white angry men“ und die Frauen, die eben auch in dem Eckensteher die zentrale Bedrohung sehen.

Mart Busche: Simona, mich würde jetzt nochmal interessieren, da wir nach Sichtbarkeiten von Räumen fragen: Wie hat die sogenannte „Flüchtlingskrise", wie hat sich durch diesen Diskurs letztendlich die Situation in den Lagern verändert oder die Wahrnehmung von Lagern in der Türkei, die türkische Grenze? Das sind ja auch alles starke Symbole.

Simona Pagano: Ich kann für mein Forschungsfeld, für Italien und die Camps für Rom_nja, die ich untersuche, sagen, dass mir auffällt, dass sich trotz der vergrößerten Sichtbarkeit von Flüchtlings- und Abschiebelagern, in Bezug auf diese Camps, so etwas wie ein schwarzes Loch auftut, in welchem diese Camps, aber auch der Rassismus gegen Rom_nja verschwinden. Denn die Camps für Rom_nja fallen noch viel zu sehr aus den öffentlichen Debatten um Lager heraus, wobei sich dies aus wissenschaftlicher Sicht langsam ändert. Und diese Unsichtbarkeit, obwohl die Camps hypersichtbar sind, ist interessant, und gerade für Rom.

Ende 2014 explodierte regelrecht ein riesiger Korruptionsskandal, als herauskam, dass Politiker_innen und Beamte an diesen Roma-Camps sowie an Flüchtlingslagern verdient haben, indem sie bestochen wurden, um das Management dieser Orte an eine bestimmte Organisation zu übertragen. Ein Teil dieser Gelder war beispielsweise für Instandssetzungsarbeiten gedacht. Wenn man in die Camps geht, sieht man, dass da kein Euro hingeflossen ist. Es kam jedoch in Bezug auf die Roma-Camps nur zu wenig Empörung, bspw. was die Verantwortlichkeiten der Institutionen für den Zustand dieser Orte angeht. Vielmehr wurden bisweilen die Rom nja selbst für diese Situation verantwortlich gemacht. Dass die Bewohner_innen selbst Geschädigte waren, drang überhaupt nicht durch. Es ist ein bisschen so, als handelte es sich um eine Blackbox beim Thema Camps für Rom_nja.

Dabei handelt es sich bei diesen Camps durchaus um lagerähnliche Strukturen, die aber aus der Kategorie „Flüchtlingslager“ herausfallen. Dies liegt meines Erachtens am Entstehungsnarrativ dieser Orte, die in den 1980er Jahren auf der Grundlage von sogenannten Gesetzen zum „Schutz nomadischer Kultur" eingerichtet wurden. Die Annahme dabei war, dass es sich bei Rom_nja und Sint_eza um Nomaden handelte, die „kulturspezifische“ Wohnmöglichkeiten benötigten. Gleichzeitig wurden sie als „Naturvolk“ 
imaginiert, das in diesen Camps bzw. sog. Halteplätzen erzogen werden sollte, im Sinne eines ,,social uplift“. Die Camps wurden in diesem Zusammenhang wie Pufferzonen verstanden zwischen der ,zivilisierten“ Mehrheitsgesellschaft und dieser ,ungewöhnlichen Kultur“.

Interessant finde ich an der Debatte, wie sie in Italien geführt wird, dass es zu einer Fixierung von Rom_nja als „Nomaden“ kommt. Dabei fällt sehr häufig unter den Tisch, dass auch Rom_nja Flüchtlinge sind bzw. sein können. Denn viele der Bewohner_innen dieser Camps sind Flüchtlinge aus den Jugoslawienkriegen. Unter dem Etikett der Nomaden sind sie jedoch gewissermaßen an diese Orte verbannt worden. Bis heute, also 20 Jahre nach Ende des Krieges, haben auch viele zum Teil noch immer keine Dokumente und sind de facto staatenlos.

Ines Kappert: Von wie vielen Menschen sprechen wir da?

Simona Pagano: Für Rom schwanken die Zahlen bzw. gibt es keine genauen Zahlen. Es wird angenommen, dass 3.000 bis 8.000 Menschen in diesen Camps wohnen. Und die Anzahl der staatenlosen beträgt für Italien ca. 15.000 .

Felix Krämer: Das hatte uns interessiert, gerade in puncto Sichtbarkeit, wie über diese ganzen Metaphern, beispielsweise Flüchtlingsströme, in den Medien bei weitem nicht alle Leute erfasst sind, die irgendwie in Lagern leben. Vielmehr verteilt sich mediale Aufmerksamkeit ungleich, wird von politischen Argumenten gelenkt. So entsteht dann dieses, wie du gerade gesagt hast, schwarze Loch, also eine Unsichtbarkeit, fast wie eine Sogwirkung, die sich wiederum manifestiert - auch räumlich.

Simona Pagano: Und bemerkenswert ist auch, dass es keine positive, affektive Reaktion gibt, das heißt, ähnlich der Solidaritätsbekundungen und Unterstützung für Geflüchtete, die es in Italien auch gibt. Das zeigt sich, was Rom_nja angeht, nur sehr wenig.

Mart Busche: Ich wollte gerade nochmal anfügen, da finden sich auch Anschlussstellen zu dem, was du gesagt hast, Ines, nicht nur in den sprachlichen Bildern: Es gibt einerseits die „Flüchtlingsströme“ und dann gibt es andererseits die „Eckensteher“. 
Ines Kappert: Ja, und es gibt die Lager, genau auch in dieser Differenz. Aber ich wollte jetzt nur noch mal ergänzen: Das Lager hat ja auch eine ganz lange Geschichte der Entmenschlichung. Es ist der Ort, an dem Menschen alle Bürgerrechte genommen werden. Sie haben kein Recht auf Mitbestimmung, sie haben kein Recht auf Arbeit, sie haben kein Recht auf Privatheit. Und das wäre eine Debatte wert, sich genau mit den Lebensbedingungen auseinanderzusetzen, die da strukturell Menschen aufgezwungen werden. Ich habe letztens mit Dilek Kolat, der Senatorin für Migration und Arbeit in Berlin gesprochen. Sie war ganz glücklich darüber, dass sie jetzt doch einen Antrag gestellt hat, dass es in den Berliner Lagern eine Auflage gibt, Schlösser einzubauen in den Toiletten. Die Idee von Lagern, dass es eben keinerlei Privatsphäre und Intimsphäre gibt, kann so weit gehen - muss nicht - aber geht sehr, sehr oft soweit, dass eben auch die Toiletten nicht abschließbar sind, weil die Subjekte jederzeit der Kontrolle zugänglich sein müssen. Sie haben jetzt diese Debatte über Schlösser im Zusammenhang mit Heimen nur für Frauen geführt. Da kam das dann mal auf oder überhaupt im Zusammenhang mit Gewalt, die in den Lagern und in den Massenunterkünften auch gegen Frauen ausgeübt wird, in dem Moment, wo man dann wieder den geflüchteten Mann, „,den Flüchtlingsmann“ in Verantwortung nehmen kann. - Es passiert wirklich Gewalt, keine Frage, es geht nicht darum, die vorhandene Gewalt in irgendeiner Weise zu verniedlichen! Die Frage ist: Wann wird das als Problem überhaupt wahrgenommen? In dem Moment, wo man den nichtweißen Täter hat. Dann wird neu über die Möglichkeit nachgedacht, dass man vielleicht die Toiletten abschließbar machen sollte.

Mechthild Bereswill: Ich würde gerne genau an der Stelle nochmal eine raumtheoretische Perspektive einbringen, denn aus einer sozialwissenschaftlichen, wie auch im engeren Sinne soziologischen Perspektive, sind die Lager totale Institutionen. Dazu gibt es Forschung und es gibt auch insbesondere aus der Gefängnisforschung natürlich Befunde, die sich nicht eins zu eins auf die Räume übersetzen lassen, über die wir hier jetzt gerade sprechen und nachdenken. Aber ich würde schon doch auch teilen, dass es bestimmte Struktureigentümlichkeiten dieser totalen Institutionen - der Lager - gibt. Du hast es genau gerade schon beschrieben: Es gibt keine abgegrenzten Privaträume. Die Privaträume sind natürlich auch schon eine Raumkonstruktion von Privatsphäre. Aber die totale Institution ist genau die Antwort darauf, indem sie als sanktionierende Institution oder eben verwahrende Institution 
genau alles unter einem Dach und unter einer Autorität verwaltet. In der Gefängnisforschung gibt es dafür das sprechende Bild: ,processing people“. Das ist ein sehr markantes Bild, weil es tatsächlich so eine doppelte Bildhaftigkeit hat von „Menschen verwalten“, sie aber auch durch diesen Lageralltag zu schleusen. Es ist also wirklich auch ein „Schleusen-Bild“. Johan Galtung hat ja den Begriff der strukturellen Gewalt auf der Basis seiner eigenen Gefängniserfahrung entwickelt. Er hat ja Wehrdienst verweigert, war inhaftiert in Norwegen und hat sich genau vor dem Hintergrund dann begonnen, mit struktureller Gewalt auseinanderzusetzen. Der Begriff ist vielfach als zu weit kritisiert worden - wir müssen das jetzt gar nicht weiter aufgreifen. Aber es gibt Forschungsbefunde und deshalb würde ich auch nochmal unterstreichen, was du gesagt hast: Es ist erstaunlich, wie wenig in dieser angeblichen Wissensgesellschaft bestimmte Wissensbestände tatsächlich aktiviert werden können, über die wir verfügen. Und das ist jetzt nicht so ein völlig anwendungsabgewandtes Grundlagenwissen, zudem gibt es genügend Menschen, die Praxiserfahrungen im Umgang mit diesem ,processing people“ haben. Es kommt aber niemand auf die Idee, diese Leute mal zu fragen, ob diese Art der Unterbringung kontraproduktiv ist, sondern sie wird einfach installiert. Und der Dezentralisierungsdiskurs ist natürlich genau daran geknüpft: Lager sind Zentralisierungsmechanismen - das ist dann quasi genau die räumliche Gegenbewegung.

Ines Kappert: Und warum eine zentrale Unterbringung? Warum wurde das auch in Deutschland eingeführt? - Damit man die Leute leichter abschieben kann, weil man in dem Moment, wo sie in die Zivilgesellschaft diffundieren, nicht mehr weiß, wo sie sind und sie schwieriger abzuschieben sind. Das ist erstmal der Grund für diese Inhaftierung oder Fixierung und Verwahrung.

Mechthild Bereswill: ... und Unterbringung, es ist eine Unterbringung.

Ines Kappert: Da ist es interessant, dass das Thema Verletzbarkeit an der Stelle keine Rolle mehr spielt. Das Konzept wird da einfach nicht mehr angewandt. Und das Problem wird auch in Deutschland wieder massiv. In den großen Flüchtlingslagern in Jordanien, in der Türkei, auf dem afrikanischen Kontinent als angewandtes Organisationsprinzip, dass man Geflüchtete autorisiert, gewisse Organisationsstrukturen zu übernehmen. Das ist undenkbar für die deutsche Regierung. Es ist undenkbar, dass man zum Beispiel die 
Lagerküche organisieren lässt von Frauen mehrheitlich, die dort gerade leben - ohne dass dies zwangsläufig Frauen sein müssten.

Mechthild Bereswill: In bundesdeutschen Gefängnissen ist das ganz üblich, dass Gefangene in die Versorgung und Herstellung von Nahrung, also in die Verteilung, ins Kochen, also in diese Prozesse mit einbezogen werden, immer unter autoritären Bedingungen. Aber trotzdem ist das natürlich ein markantes Beispiel und mir geht es nun nicht darum zu sagen: „Jetzt schauen wir mal in den Strafvollzug und dann haben wir irgendwie bessere Strukturen“, sondern es gibt da Struktureigentümlichkeiten, die sich ähneln. Es gibt da Analogien. Und aus denen kann auch gelernt werden, weil wir aus räumlichen Bedingungen von geschlossenen oder teilgeschlossenen Unterbringungen in großen Gruppen wissen, dass sie tatsächlich gewaltförmige Interaktionen befördern. Das soll nicht als eine deterministische Sicht auf Institutionen und Handeln verstanden werden, sondern das sind tatsächlich institutionelle Strukturen, die Möglichkeitsräume öffnen für Agency. Und diese Agency ist unter anderem auch auf Gewalt bezogen und das macht auch Sinn, das ist nachvollziehbar, das können wir verstehend rekonstruieren und dann sind das sozusagen vernunftgeleitete Handlungsentscheidungen, zu sagen: „Ich drohe Gewalt an, um hier eine bestimmte Position zu erlangen“. Und meine große Sorge ist, was ich insbesondere zu Beginn der Situation, dieser dramatisierten Situation, beobachtet habe, dass im Diskurs das Ganze kulturalisiert wird. Etwa, dass es, wenn Menschen sich schlagen bei der Essensausgabe, was damit zu tun hätte, dass sie sich irgendwie religiös nicht grün sind - oder ähnlich abstruse Interpretationsmuster, anstatt zu dem Naheliegenden zu kommen, dass das die Unterbringungsstruktur ist.

Felix Krämer: Vielen Dank! Wir wollen hier einen kurzen Cut machen, die Diskussion öffnen, um euch und Ihnen die Möglichkeit zu geben, die Referent_innen bzw. die Diskussionsrunde auch etwas zu fragen. Dann werden wir zum Anti-Genderismus kommen und schauen, wie wir diese Intersektion, diese Verschränkung von Raum und Geschlecht, Geschlechterverhältnissen und sexuellen Abgrenzungen mit reindiskutieren können in diese spannenden Fragen nach räumlichen Verteilungen und Zuschreibungen. ${ }^{3}$

3 An der Stelle fand eine Diskussion mit Fragen aus dem Publikum an die Referent_innen auf dem Podium statt. 


\section{GESCHLECHT-MACHT-RAUM: ANTI-GENDERISMUS}

Felix Krämer: Der nächste Part, in dem wir uns dem Anti-Genderismus zuwenden wollen, ist auch ein Bereich, in dem gesellschaftlich in sozialen oder auch in symbolischen Räumen eine bestimmte Bewegung vonstatten geht, die etwas zu tun hat mit diesen Diskursen, die wir angesprochen haben - also mit der Frage: Wer wird in welche geschlechtliche Position, in welches Gewaltverhältnis gesetzt?

Mart Busche: Ich meine, es geht in der Diskussion um einen öffentlichen Artikulationsraum oder um einen politischen Artikulationsraum. Eigentlich wäre für mich die Frage da nochmal: Welche Verteilungskämpfe finden sowohl in der Diskussion, wie wir sie gerade hatten um Rassismus, und jetzt auf das Feld des Anti-Genderismus bezogen, statt. Genderismus ist ein Begriff von Erving Goffman, der gewissermaßen enteignet wurde. Manche streiten wieder dafür, wir sollten uns den wiederholen, doch das ist vielleicht gar nicht so wichtig. Wir haben jedenfalls gestern gehört, dass Alice Schwarzer schon damals so eine Art Shitstorm abgekriegt hat. Immer wenn Feminist_innen an die Öffentlichkeit gehen, kriegen sie sowas ab, und jetzt hatten wir es 2014, dass dann auch mal Professor_innen angegriffen wurden, also eine relativ hohe Statusgruppe. Und die Blogger_innen, Jasna Strick ist ja nicht hier, die kriegen das ja auch schon eine ganze Weile ab, das wissen wir ja auch. Was wären da eigentlich Perspektiven, um diese Kämpfe möglicherweise zu verknüpfen oder wo sind die Sphären total getrennt, wo könnte man auch zusammenkommen? Das werfe ich jetzt mal in die Runde, weil ich natürlich weiß, dass sehr viele Akademiker_innen in diesem Raum sind, die sich damit auch schon beschäftigt haben.

Felix Krämer: Gibt es Möglichkeiten, diese unterschiedlichen Ebenen, wenn es überhaupt Räume sind, zu verknüpfen? Diesen Feminismus 2.0, 3.0, 4.0 es sind ja viele Ebenen da, die sich auf Öffentlichkeitspolitiken beziehen, wo auch immer die Realität stattfindet, wo unterschiedliche Artikulationsmöglichkeiten existieren, von denen man aber nicht genau weiß, wie kriegt man da Zugriff. 
Ines Kappert: Es gibt ja einen Ansatz in der feministischen Netzpolitik, Gesetzesreformen zu fordern, mit der Begründung, dass der Tatbestand der digitalen ,hate speech“ einfach noch nicht übersetzt worden ist in den juristischen Diskurs. Das ist noch ziemlich am Anfang. Die Ambivalenz, mit der wir es da zu tun haben, ist, dass wir auf der einen Seite zwar Schutzräume im Netz brauchen, aber das Netz ja nicht zum Überwachungsraum machen wollen. Und das ist, glaube ich, die große Frage und da gibt es momentan, wenn ich das richtig sehe, ich lasse mich da gern eines Besseren belehren, noch keine Lösung. Das fängt gerade erst an. Ich finde, das ist alles noch ziemlich in der Lamäng. Was aber jetzt anfängt, ist, dass es jetzt einen Austausch gibt von Mails, die man zurückschickt, wenn man eben Betroffene von hate speech wird. Wo dann eben bestimmte Paragraphen genannt werden und die Polizei sich darum kümmern würde und in manchen Fällen werden jetzt tatsächlich erstmals polizeiliche Ermittlungen in Gang gesetzt, in dem Sinn, dass die Person einen Brief bekommt. Das ist eine neue Entwicklung, denn das wurde bislang überhaupt noch nicht, bis auf einige wenige Ausnahmen, in der Breite als Straftatbestand verfolgt. Ich meine, dass man in die Richtung weiterdenken muss. Das steht aber noch am Anfang, wie man genau Überwachung verhindert und Schutzräume schafft.

Mechthild Bereswill: Ich würde da nochmal aus dem Blickwinkel der Sozialforscherin darauf antworten. Ich habe bestimmte Texte, die kursieren, aus diesem Anti-Genderismus, der sich ja auch verknüpft mit rassistischen Argumentationsfiguren, mit Studierenden gemeinsam untersucht, im Zuge der Lehre. Und das ist jetzt ja nur ein Beispiel, mit dem ich sagen will, ich gehe nicht davon aus, dass wir alle immer in der Lage sind, die Verknüpfpunkte in diesen Diskursen zu erkennen, sondern dass das auch eine Analysearbeit ist, dass diese Verknüpfpunkte auch untersucht werden sollten. Umgekehrt, wenn Sie nach der Politik fragen, Sie haben ja Laclau schon genannt, könnte ich mit der Chantal Mouffe sagen, dass so eine gemeinsame Analyse durchaus auch dann deutlich macht, wo die Äquivalenzpunkte sind, wo zunächst ganz unterschiedliche Interessen oder Bezüge oder Zugehörigkeiten dann trotzdem auch zu Bündnissen führen. Das ist eben tatsächlich, glaube ich, ein ganz, ganz kleinschrittiger und zähflüssiger Prozess. Und wenn ich als Wissenschaftlerin darauf antworte, denke ich, meine Aufgabe ist da irgendwie eine Analyse auch mit zu betreiben - ohne, dass daraus sofort politisches Handeln wird. Da würde ich mich eher auch etwas stärker zurücknehmen 
und mit Elias von „Engagement und Distanzierung“ sprechen, denn Soziologie ist stark verwickelt in die aktuelle Situation, in der sie Soziologie oder Sozialwissenschaft betreibt. Aber die Stärke von Forschung ist genau an der Stelle, dass wir ein Stück auf Distanz gehen und dadurch zum Beispiel sehen, dass in einem Papier, von dem wir zunächst denken, es enthalte wirre Argumente, sich dann in einer rekonstruktiven Perspektive eben auch bestimmte Argumentationsfiguren zeigen. Dieser Mühsal müssen wir uns unterziehen. Ich finde das schwierig. Ich lese dieses Zeug nicht gerne und würde da auch lieber nicht drüber sprechen oder arbeiten, aber ich glaube, wir müssen das irgendwie durchqueren. Wer auch immer „Wir“ hier ist, das war jetzt auch schon wieder affirmativ: Alle, die meinen, dass sie sich damit in irgendeiner Weise aktiv und produktiv mit so einer Grenze von Wissenschaft, Forschung und politischer Einmischung auseinandersetzen.

Mart Busche: In Berlin hat Lann Hornscheidt, eine Person mit Professur für Linguistik eine E-Mail-Adresse eingerichtet - weil sich diese Person unter anderem für neue Pronomina einsetzt und das fanden viele nicht gut - und die heißt hatemails(at)lannhornscheidt. Da konnten alle Leute ihren Shit sozusagen abladen und die Leute haben das tatsächlich gemacht: Das ist sicher ein reiches Archiv für solche Analysen. Meine Frage ging schon auch Richtung Praktiken. Ich meine, Wissenschaftler_innen auf hohem Niveau haben Fachgesellschaften, die ihnen dann Solidaritätserklärungen schreiben. Das habe ich als kleine Blogger_in vielleicht nicht unbedingt, weil es keinen Fachverband für Blogger_innen gibt - es sind vielleicht ,Mädchenmannschaft“ oder die sogenannten „Holzmedien“, die sich damit beschäftigen, wobei auch Verschaltungen von Virtuellem und Realen stattfinden, die vielleicht auch interessant wären, im Sinne von Kampagnen (Holzmedien, möglicherweise, weil Zeitungspapier aus Bäumen produziert wurde - ich weiß gar nicht, wo der Begriff eigentlich herkommt).

Ines Kappert: Es ist natürlich auch mein Job hier als Journalistin zu sprechen, aber ich musste so ein bisschen lächeln als dann so die Gruppe der Professor_innen über Hatemails zu erschrecken begann. Als Journalistin ist man da wirklich ganz anderen Dingen ausgesetzt, gerade auch, wenn man einen Migrationshintergrund hat. Da kann man dann andere fragen. Klar, natürlich, Zeitungen haben Rechtsabteilungen, das ist natürlich hilfreich. Aber das hat dann auch nicht so viel gebracht, weil eben die Rechtslage so verworren ist, 
dass man Leute eben noch gar nicht so wirklich verklagen kann. Wenn du jetzt nach einer Praxis fragst, als Journalistin: Du liest die Kommentare einfach nicht mehr, das ist vollkommen klar. Ich als Meinungsjournalistin fand das immer ganz gut, dass diese ganzen Hassmails unter meinen Kommentaren standen, denn wenn mich jemand gefragt hat: „Gibt es wirklich Sexismus?", dann habe ich ihn auf die Kommentare verwiesen und dann musste ich nichts mehr sagen. Aber natürlich kann man das nicht lesen, weil das verletzend ist und dann wird das eben ignoriert. Dann muss man aushandeln mit den Online-Redaktionen, welche Kommentare sie löschen - Kriterien aushandeln. Aber diese Antwort ist natürlich normativ völlig unbefriedigend. Das ist ein rein empirisches Rantasten.

Felix Krämer: Wir kommen langsam zum Schluss. Ich würde gern Simona noch eine Frage stellen, die das vielleicht zusammenbindet, was wir zu Beginn diskutiert hatten, wie sich Frauen- und Geschlechterforschung auch in ihrer intersektionalen Ausrichtung in den letzten Jahrzehnten entwickelt hat. Ich erinnere mich, dass wir mit Gudrun-Axeli Knapp, die auch Gastdozentin am Kolleg war, als das Genderzentrum in Göttingen gegründet wurde, darüber diskutierten und da eine Art Standortbestimmung, eine Art Verortung versucht haben, entlang der Frage, in welchem politisch-diskursiven Bereich wir uns in der Diskussion befinden. Da kam auch die Ambivalenz zwischen Gender Studies und Queer Studies als politische Intervention zur Sprache. Wir haben über hegemoniale Geschlechtermatrizen, in die man sich einsortieren muss, auch als Forschungsprogramm, geredet und eben auch über den Topos Diversitätsforschung diskutiert. Daher möchte ich dich nochmal fragen, wie deine Erfahrungen mit Intersektionalität sind, speziell wenn Gender und Rassismus zusammengebracht werden.

Simona Pagano: Meine Erfahrung ist zunächst so, dass Gender immer noch eine Kategorie ist, die dazugefragt, dazuaddiert wird und werden muss. Es ist nicht selbstverständlich, dass Gender Teil von Forschungen zu Rassismus beispielsweise ist. Dabei stellt sich eben auch die Frage, wovon wir sprechen, wenn wir von Gender sprechen, dass Genderforschung nicht gleichbedeutend mit einer feministischen Forschung sein muss. Ebenso wenig wie diese Forschung dann auch intersektional sein muss. Aus meiner Forschung kann ich sagen, dass aber eigentlich gerade die Verknüpfung von Rassismus und 
Sexismus oder eine vergeschlechtlichte Perspektive zum Beispiel auf Rassismus gegen Rom_nja fruchtbar sein kann, um dieses Phänomen weitergehend $\mathrm{zu}$ verstehen. Ich versuche das erneut an einem Beispiel zu konkretisieren: 2008 wurde in Italien ein Notstand ausgerufen in Bezug auf die Anwesenheit „nomadischer Bevölkerungen“, also Rom_nja, auf italienischem Staatsgebiet. Den Aufhänger bildete ein Tötungsdelikt: Kurz zuvor hatte ein Mann aus einem Camp in Rom eine Frau überfallen und getötet. Dieses Delikt führte dazu, dass die Siedlung, in der er lebte, komplett geräumt und zerstört sowie der Notstand ausgerufen wurde und versicherheitlichende Maßnahmen eingeführt wurden, die mit Zählungen der Bewohner_innen der Camps einhergingen, Gruppenfotos und z.T. der Erfassung biometrischer Daten dieser Menschen. Zum anderen wurde dieser Notstand in einen patriarchalen Rahmen zum „Schutz“ „italienischer“ Frauen gefasst. Also auch hier wurde das Narrativ bemüht „einheimische“ Frauen vor ,ausländischen“ Männern schützen zu müssen. [TAHRIRPLATZ] Und in der Tat wurde es dann möglich, EU-Bürger_innen leichter abzuschieben.

Doch auch das Sprechen über Rassismus ist schwierig. In meiner Forschung arbeite ich unter anderem mit David Goldberg. Eine seiner Thesen lautet, dass Rassismus und das Konzept „Rasse“ mit dem Kolonialrassismus und mit dem Holocaust im Zusammenhang stehen. Das heißt folglich, dass wir uns heute nicht mehr als rassistisch verstehen, also dass wir diesen Rassismus überwunden haben. Das heißt aber auch, dass Rassismus verleugnet wird, bzw. dass er auf eine individuelle Ebene begrenzt wird. Rassismus wird als individuelle, verfehlte Einstellung betrachtet oder eben als Problem extrem rechter Gruppierungen. Rassistisch sind außerdem immer die anderen: Der Satz „Ich bin nicht rassistisch, aber“ fasst diesen Rassismus ganz gut zusammen. Diese Verleugnung, wie sie David Goldberg nennen würde, führt dazu, dass rassistische Äußerungen, Praktiken etc. verbreitet werden, ohne dass diese als solche benannt werden. In der Folge kommt es zu einer Depolitisierung und Dethematisierung von Alltagsrassismus aber auch von Antirassismus. Stattdessen wird Diversität zelebriert, Inklusion, Interkulturalität usw. - während strukturelle Macht- und Ungleichheitsverhältnisse zugunsten eines glattgebügelten Pluralismus verschwiegen werden. Diese neue Konjunktur des Rassismus benennen Alana Lentin und David Goldberg als postracial, also als postrassistisch. Diese postrassistische Rahmung kann man wiederum in einen größeren neoliberalen Zusammenhang stellen. In der Fokussierung auf individuelle Fähigkeiten, der (Un-)Fähigkeit sich selbst zu 
vermarkten, das Beste aus sich zu machen, geraten strukturelle Dimensionen aus dem Blick. Ein Scheitern ist dann selbstverschuldet. Oder eben so: Die eigene Kultur ist schuld, die eigene Ethnizität ist schuld, das eigene Geschlecht ist schuld. Und so ähnlich gestaltet es sich in meinem Forschungsfeld: Es aus dem Camp herauszuschaffen, liegt ebenfalls in der eigenen Verantwortung der Bewohner_innen der Camps. Wer es nicht schafft, ist eben im Grunde selbst schuld.

Um nochmal auf deine Frage zurückzukommen, würde ich sagen: Ich sehe, dass es durchaus einen Bedarf gibt an einer intersektionalen Forschung, aber einer intersektionalen Forschung, die eben Machtverhältnisse untersucht, also deren Verschränkung wie es beispielsweise Rassismus und Sexismus sind. [EINLEITUNG]

Felix Krämer: Wir bedanken uns recht herzlich bei Mechthild Bereswill, bei Simona Pagano für die Präsentation ihrer Überlegungen, bei Ines Kappert, dass sie zu uns gekommen ist heute und mit uns diskutiert hat und bei Ihnen, dass Sie dagewesen sind, uns ihr Ohr und Ihren Geist geschenkt haben 